\title{
A new approach on lithium-induced neurotoxicity using rat neuronal cortical culture: Involvement of oxidative stress and lysosomal/mitochondrial toxic Cross-Talk
}

https://doi.org/10.1515/mgmc-2020-0003

Received November 24, 2019; accepted March 04, 2020.

\begin{abstract}
Lithium (Li) is a widely-used medication for the treatment of patients with bipolar disorder. Li causes different complications. One of the most important adverse effects of $\mathrm{Li}$ is neurotoxicity. Neurotoxicity is usually irreversible which may lead to very important complications. The symptoms of Li-induced neurotoxicity include tremor, delirium, seizures, coma, and death. In this study, we wanted to evaluate the exact sub-cellular mechanisms of Li-induced neurotoxicity.

For this purpose, we used primary neuronal cortical culture for investigating lithium-induced neurotoxicity. We applied the postnatal rat pups for isolating the cortical neurons. After that, we evaluated neural viability, neural reactive oxygen specious (ROS), lipid peroxidation, mitochondrial membrane potential (MMP), lysosomal membrane integrity (LMI), and reduced (GSH) and oxidized (GSSG) glutathione.

Our results demonstrated that the cytotoxic effect of Li has mediated through lysosomal membrane leakage associated with ROS formation and reduction of MMP. Furthermore, the incubation of isolated neurons with $\mathrm{Li}$ caused rapid GSH depletion (as GSSG efflux) as another marker of cellular oxidative stress.
\end{abstract}

\footnotetext{
* Corresponding author: Jalal Pourahmad, Department of Pharmacology and Toxicology, Faculty of Pharmacy, Shahid Beheshti University of Medical Sciences, Tehran, Iran, P. O. Box: 141556153 tel: +98-2122558786, fax +98-2188209620, e-mail: j.pourahmadjaktaji@utoronto.ca

Bahareh Sadat Yousefsani, Research Institute for Islamic and Complementary Medicine, Iran University of Medical Sciences, Tehran, Iran, School of Persian Medicine, Iran University of Medical Sciences, Tehran, Iran Romina Askian, Department of Pharmacology and Toxicology, Faculty of Pharmacy, Shahid Beheshti University of Medical Sciences, Tehran, Iran
}

We concluded that Li causes neurotoxicity in a dosedependent manner. Besides, Li-induced neurotoxicity is a result of the generation of ROS and LP, which leads to mitochondrial/lysosomal toxic cross-talk.

Keywords: lithium; neurotoxicity; neuronal cortical culture; mitochondria; lysosome; oxidative stress

\section{Introduction}

Biologicals, chemicals, metals as well as medications could be considered as the neurotoxic agents (Clausen et al., 2019; Garza-Lombo et al., 2019; Horta et al., 2019; Richardson et al., 2019; Staff et al., 2019). These factors, depending on whether they affect the central or peripheral part of the brain, cause complications on the structure and function. This health issue can lead to serious problems such as memory impairment, learning difficulties, and visual and hearing impairment (Costa et al., 2020). Many efforts were conducted to find ways for preventing many diseases such as neurotoxicity; for example, the use of different types of antioxidants (Baradaran Rahimi et al., 2019; Huang et al., 2019; Ji et al., 2019; Mansoori et al., 2011; Sohrabvand et al., 2017; Yousefsani et al., 2019). Neurotoxicity is usually irreversible; therefore, it is very important to identify the causes of neurotoxicity and their mechanisms for finding ways for prevention (Ferraro et al., 2019; Kordafshari et al., 2017; Zhang et al., 2020). Lithium (Li) is a well-known medication for the treatment of patients with bipolar mood disorder. Recent researches demonstrated that Li could be helpful for the treatment of Alzheimer's disease (Hampel et al., 2019). It also could be effective for the treatment of Parkinson's disease (Arraf et al., 2012). This widely used medication has a narrow therapeutic index. This means that this medication is 
potentially toxic (Salimi et al., 2017a). Therefore, the small errors in prescription, drug interactions or patient mistakes in taking medications can lead to poisoning. Furthermore, some diseases can also increase Li plasma concentrations and cause toxicity, like impaired renal function, chronic causes of volume depletion, and infections (de Cates et al., 2017; Vodovar and Megarbane, 2017). Li is easily absorbed through the intestines, distributed in the body, and then almost completely excreted through the kidneys. Although Li neurotoxicity mainly occurs due to Li plasma levels above $1.0 \mathrm{mmol} / \mathrm{L}$, in some cases, neurotoxicity could happen in the normal plasma concentration (Megarbane et al., 2014; Mesquita et al., 2010; SorianoBarcelo et al., 2015). Therefore, it is important to note that plasma concentrations of $\mathrm{Li}$ should not only be considered but also, it is necessary to consider any clinical signs as a warning factor for the onset of toxicity. Most of the Li-induced toxicity occurs at therapeutic doses. Lethal concentrations of $\mathrm{Li}$ is above $3.5 \mathrm{mmol} / \mathrm{L}$, suggesting that hemodialysis is the only treatment (Decker et al., 2015).

The symptoms of Li neurotoxicity include tremor, delirium, drowsiness, ataxia, muscle weakness and twitching, slurred speech, pseudodementia, psychomotor slowing, disorientation, seizures, coma, and death (Soni, 2019). There are important factors considered as risk factors for Li-induced neurotoxicity. These include: hypertension, heart failure, rapid correction of hyponatremia and hyperlipidemia, neurologic illness, enteric fever, acute gastroenteritis, and epilepsy (Lang and Davis, 2002; Malhotra et al., 1985; Mani et al., 1996; Swartz and Jones, 1994).

Fever is usually one of the common signs of Li intoxication (Donaldson and Cuningham, 1983; Hansen and Amdisen, 1978). High body temperature induces protein degradation, therefore, pathologic changes would be observable in the nerve and glial cells (Hancock and Vasmatzidis, 2003). Usually, in the early stages of lithium intoxication, body temperature is normal, but as the toxicity progresses, body temperature increases. Increasing body temperature can also cause dehydration and further poisoning (Schou et al., 1968).

Researches revealed that oxidative stress production is the most important reason for Li toxicity (Salimi et al., 2017a). Li can increase the $\mathrm{Ca}^{2+}$ level in the cultured cerebellar granule neurons and causes neurotoxicity (Yao et al., 1999). Besides, Li induced lipid peroxidation (LP) in synaptosomes, which leads to the modification of synaptic endings and the lipid content of synaptoplasmatic membranes that consequently leads to severe disturbances in the function of neurotransmitter uptake systems and depolarization-dependent calcium channels (Efendiev and Kerimov, 1994; Sawas and Gilbert, 1985; Sawas et al., 1986). This can lead to depression, sleep disorders, and other important neurotoxic signs (Taranova and Nilova, 1986).

Although neurotoxicity signs of $\mathrm{Li}$ have been recognized recently, the exact sub-cellular mechanisms are still unclear. For this purpose, the rat primary neuronal cortical culture was used for evaluation of mechanisms behind the Li-induced neurotoxicity.

\section{Materials and methods}

\subsection{Chemicals}

10×Hank's balanced salt solution (HBSS), (4-(2-hydroxyethyl)-1-piperazineethanesulfonic acid) (HEPES) buffer, fetal bovine serum (FBS), neurobasal medium, minimal essentialmedium(MEM) with Earle'ssalts and L-glutamine, penicillin-streptomycin, gluta MAX-I supplement, B27 serum-free supplement, trypsin, cell-freezing medium from was purchased Invitrogen (Burlington, ON). Sodium pyruvate, $\mathrm{Li}^{+}$carbonate, D-glucose, selenium dioxide, putrescine, progesterone, bovine transferrin, insulin from Sigma (Oakville, ON, Canada).

\subsection{Animals}

All experiments and procedures in this study were performed in full compliance with the NIH Guide for the Care and Use of Laboratory Animals guidelines for animal research and Shahid Beheshti University of Medical Sciences Animal Ethics Committee. Experiments were held on young (1-day-old pups) male Wistar rats.

\subsection{Isolating the neurons}

Isolating the neurons were done based on Meberg and Miller method (Meberg and Miller, 2003). Under a laminar flow hood, the brains of postnatal rat pups were removed and placed in a dish containing CMF-HBSS (calcium-, magnesium-, and bicarbonate-free Hank's balanced salt solution). Under a microscope, the cerebral hemispheres and hippocampus were removed carefully and transferred to a drop of CMF-HBSS and chopped the tissues as finely as possible and then transferred to a $50 \mathrm{~mL}$ conical centrifuge tube in a final volume of $12 \mathrm{~mL}$ CMF-HBSS containing $1.5 \mathrm{~mL}$ each of $2.5 \%$ trypsin and $1 \%$ (wt/vol) DNase and 
incubated in a $37^{\circ} \mathrm{C}$ water bath for $5 \mathrm{~min}$. After triturating the cell suspension, the chunks of undissociated tissue were removed by passing through a cell strainer. The entrifugation was done (10 $\mathrm{min}, 1000 \mathrm{rpm}$ ) to remove the enzymes and lysed the cells and the supernatant was discarded. Hemocytometer was used to calculate cell density. Cells were suspended with the Glial Medium (430 mL DMEM, $50 \mathrm{~mL}$ FBS, $5 \mathrm{~mL}$ penicillin, $5 \mathrm{~mL}$ sodium pyruvate) and the cell density was adjusted to $10^{6}$ cells $/ \mathrm{mL}$ in the flasks. Each flask contained $10 \mathrm{~mL}$ of isolated neurons suspension. The neurons were used for toxicological tests after one day of incubation (Yousefsani el al., 2020).

Before performing each test, we centrifuge the cell culture (1 $\mathrm{min}, 1000 \mathrm{rpm}$ ) to separate cells from the culture medium and other substances in it. Isolated neurons were separated in the same volume of incubation buffer (Krebs $750 \mathrm{~mL}$ and HEPES $2.25 \mathrm{~g}, \mathrm{pH}$ 7.4). This was done to reduce interference (Pourahmad et al., 2011).

\subsection{The viability of neurons}

The trypan blue $(0.2 \% \mathrm{w} / \mathrm{v})$ exclusion test was performed for determination of the survival of isolated neurons with the intactness of the plasma membrane. Taking the samples of the incubated neurons was done at different time points during $24 \mathrm{~h}$ (Pourahmad and O’Brien, 2000).

\subsection{Determination of reactive oxygen species}

Dichlorofluorescindiacetate (DCFH-DA, 1.6 $\mu \mathrm{M}$ ) was used for determination of Li-induced ROS formation. $3 \mathrm{~mL}$ of isolated neurons suspension $\left(3 \times 10^{6}\right.$ cells $\left./ \mathrm{mL}\right)$ were centrifuged ( $1 \mathrm{~min}, 1000 \mathrm{rpm}$ ) then, the supernatant was discarded. The isolated neurons were incubated in the test tube containing dye $\left(10 \mathrm{~min}, 37^{\circ} \mathrm{C}\right)$. DCFH-DA penetrated isolated neurons and hydrolyzed to non-fluorescent dichlorofluorescein (DCFH). The fluorescence intensity of DCF was measured using a Shimadzu RF5000U fluorescence spectrophotometer. Excitation and emission wavelengths were $500 \mathrm{~nm}$ and $520 \mathrm{~nm}$, respectively. The results were expressed as fluorescent intensity per $10^{6}$ cells (Shen et al., 1996).

\subsection{Lipid peroxidation assay}

The amount of TBARS (thiobarbituric acid-reactive substances) was measured during the decomposition of lipid hydroperoxides. $3 \mathrm{~mL}$ phosphoric acid (1\%) and $1 \mathrm{~mL}$ thiobarbituric acid (0.6\%) was added to $0.5 \mathrm{~mL}$ of centrifuged neurons (1 $\mathrm{min}, 1000 \mathrm{rpm})$. After that the mixture was heated in a boiling water bath ( $45 \mathrm{~min}) .4 \mathrm{~mL}$ of $\mathrm{n}$-butanol was added to the mixture and centrifuged (10 min, $5000 \mathrm{rpm}$ ) after cooling. The organic layer was transferred to a quartz cuvette. The PG Instruments T60V spectrophotometer was used for measuring the absorbance (Khansari et al., 2017).

\subsection{Intracellular GSH and extra cellular GSSG assessment}

The spectrofluorometric method was used for evaluation of GSH and GSSG (Hissin and Hilf, 1976). In this method a reaction between orthophetaldehyde (OPT) $(1 \mathrm{mg} / \mathrm{mL})$ and GSH (in $\mathrm{pH}=8$ ) and GSSG (in $\mathrm{pH}=12$ ) in incubation buffer (Krebs $750 \mathrm{~mL}$, HEPES $2.25 \mathrm{~g}$, pH 7.4) formed a fluorescent substance. Therefore, for evaluating GSH, after centrifugation (1 min, $1000 \mathrm{rpm}$ ) of $1 \mathrm{~mL}$ of neuron suspension, the culture medium was excluded. Then, $1 \mathrm{~mL}$ trichloroacetic acid (10\% w/v) was added to the neurons and centrifuged (10 $\mathrm{min}, 5000 \mathrm{rpm}$ ) again. $4.4 \mathrm{~mL}$ of incubation buffer $(\mathrm{pH}=8)$ was added to $0.5 \mathrm{~mL}$ of supernatant. After that, $100 \mu \mathrm{L} \mathrm{OPT}(1 \mathrm{mg} / \mathrm{mL})$ was added to the above-mentioned solution and left for $15 \mathrm{~min}$ (at room temperature) and then transferred to a quartz cuvette. For evaluating GSSG, $200 \mu \mathrm{L}$ of N-ethylmaleimide $(0.04 \mathrm{~N})$ was added to $0.5 \mathrm{~mL}$ of supernatant to prevent oxidation of GSH to GSSG. $4.2 \mathrm{~mL}$ of incubation buffer $(\mathrm{pH}=12)$ was added to this mixture, after that, $100 \mu \mathrm{L}$ OPT $(1 \mathrm{mg} / \mathrm{mL})$ was added to the above-mentioned solution and left for $15 \mathrm{~min}$ (at room temperature). Then, it was transferred to a quartz cuvette. Each sample was measured using a fluorometer (350 $\mathrm{nm}$ excitation and $420 \mathrm{~nm}$ emission wavelengths) (Salimi et al., 2017b).

\subsection{MMP assay}

Mitochondrial uptake of the cationic fluorescent dye, rhodamine123, has been used for estimation of MMP (Kiani et al., 2017). For exclusion the culture medium, $0.5 \mathrm{~mL}$ of neuron suspension was centrifuged (1 min, $1000 \mathrm{rpm}$ ) and then $2 \mathrm{~mL}$ of rhodamine123 $1.5 \mu \mathrm{M}$ was added to the cells. After $10 \mathrm{~min}$ of incubation $\left(37^{\circ} \mathrm{C}\right)$, the suspension was centrifuged (1 min, $1000 \mathrm{rpm}$ ) again. Then, the supernatant was transferred to a quartz cuvette. Each sample was measured using a fluorimeter $(490 \mathrm{~nm}$ 
excitation and $520 \mathrm{~nm}$ emission wavelengths). Our data were shown as the percentage of MMP collapse $(\% \Delta \Psi \mathrm{m})$ in all groups.

\subsection{LMI assay}

Isolated neuron lysosomal membrane stability was evaluated by redistribution of the fluorescent dye, acridine orange (AO) (Pourahmad et al., 2005). For exclusion the culture medium, $0.5 \mathrm{~mL}$ of neuron suspension was centrifuged (1 min, $1000 \mathrm{rpm})$ and then, $2 \mathrm{~mL} \mathrm{AO} \mathrm{(5 \mu M)}$ was added to the cells. After $10 \mathrm{~min}$ of incubation $\left(37^{\circ} \mathrm{C}\right)$, the suspension was centrifuged (1 $\mathrm{min}, 1000 \mathrm{rpm}$ ) again. Then, the supernatant was transferred to a quartz cuvette. The absorbance was measured using a fluorimeter ( $495 \mathrm{~nm}$ excitation and $530 \mathrm{~nm}$ emission wavelengths). Our data were shown as the percentage of lysosomal membrane leakiness in all groups.

In all tests, $\alpha$-Tocopherol succinate as lipid antioxidant, mannitol as hydroxyl radical scavenger, carnitine as MPT pore sealing agents and chloroquine as lysosomotropic agent was added to the isolated neuron culture $1 \mathrm{~h}$ before Li administration. These concentrations were chosen based on previous researches (Pourahmad et al., 2011; Yousefsani et al., 2018). None of these protective agents showed any toxic effects or interaction on isolated neurons or experiments at concentrations used (data not shown).

\subsection{Evaluation of apoptosis}

For quantification the percentage apoptosis versus necrosis by flowcytometric analysis (Cyflow Space-Partec), Annexin V fluorescein isothiocyanate (FITC)/propidium iodide (PI) stained cells was applied. Annexin V/PI assay was done using a commercial kit (Immunotech; Beckman Coulter, Dubai, United Arab Emirates) according to the manufacturer’s instructions (Salimi et al., 2015).

\subsection{Statistical analysis}

The statistical analysis was done using the Graph Pad Prism software, version 8.0.2 (Graph Pad Software, San Diego, CA, USA). Data are reported as mean \pm standard deviation of three separated tests. Data were analyzed using one-way analysis of variance followed by TukeyKramer test as the post hoc test. The minimal level of significance chosen was $\mathrm{p}<0.05$.

\section{Results}

\subsection{Effect of Li on cell viability in isolated neural culture}

As shown in Figure 1, Li-treated neurons after $2 \mathrm{~h}$ showed a significant decrease in cell viability in a dose-dependent manner ( $\mathrm{p}<0.001)$. IC50 for Li after $2 \mathrm{~h}$ exposure was $1.98 \pm 0.87 \mathrm{mM}$.

\subsection{Effect of Li on ROS formation in isolated neural culture}

As shown in Figure 2, Li produced a marked increase in ROS generation $(p<0.001)$ in a dose-dependent manner.

\subsection{Effect of Li on lipid peroxidation in isolated neural culture}

As demonstrated in Figure 3, Li significantly increased the TBARS $(\mathrm{p}<0.001)$ in a dose-dependent manner.

\subsection{Effect of Li on MMP in isolated neural culture}

Because of neuronal ROS formation, Li induced a rapid decline of MMP, an apparent marker of mitochondrial dysfunction $(\mathrm{p}<0.001)$ (Figure 4).

\subsection{Effect of Li on LMI in isolated neural culture}

When the isolated neurons were loaded with a lysosomotropic agent (acridine orange), severe oxidative damage to lysosomal membrane demonstrated a significant $(p<0.001)$ release of acridine orange into the cytosolic fraction ensued within $2 \mathrm{~h}$ of incubation with $\mathrm{Li}$ (Figure 5).

\subsection{Effect of Li on intracellular GSH and efflux GSSG}

As demonstrated in Figure 6, incubation of neurons with Li caused another marker of cellular oxidative stress, rapid neural glutathione (GSH) depletion. Most of the Li-induced 


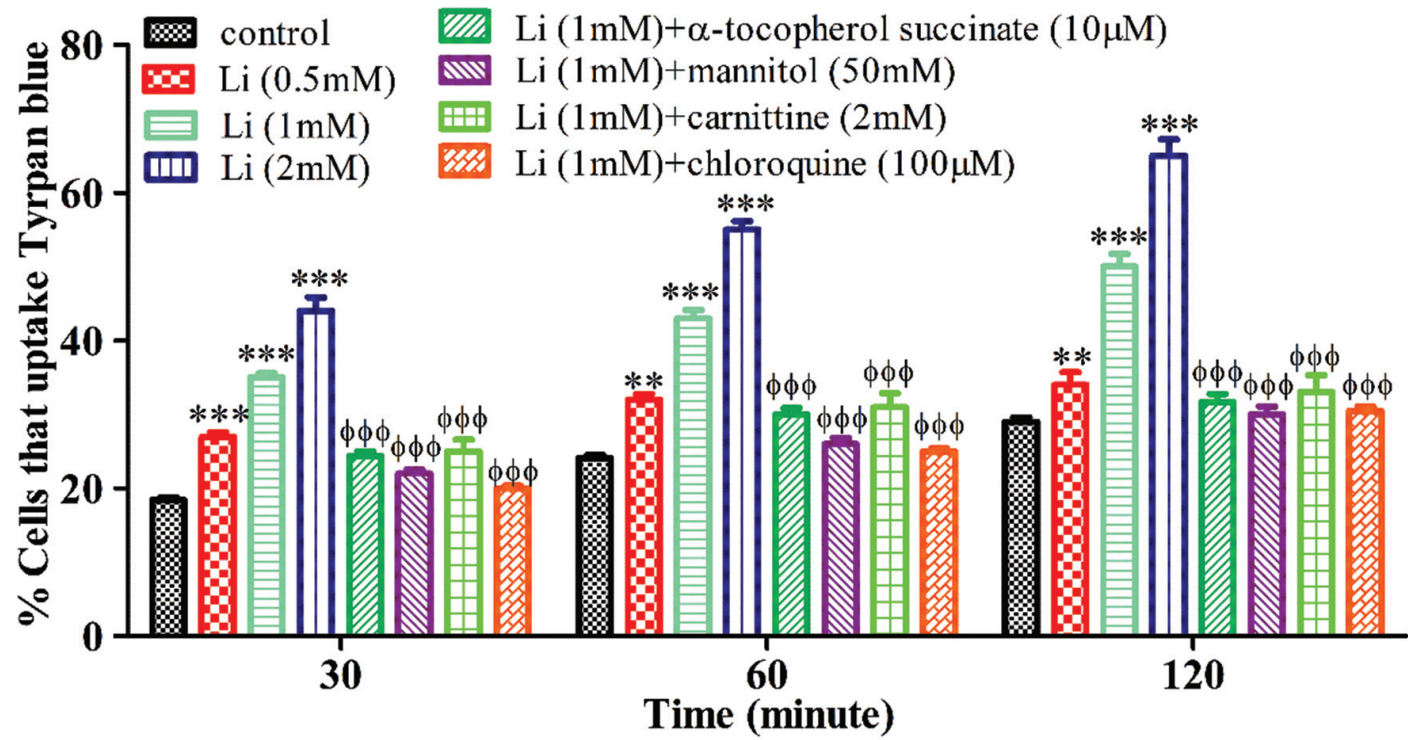

Figure 1: Effect of different concentrations of Li on neuronal death using primary neuronal cortical culture. Determination of cytotoxicity was done as the percentage of cells that absorb trypan blue. Values are shown as mean \pm SD of three separate experiments $(n=3)$.

${ }^{* \star} p<0.01,{ }^{* \star *} p<0.001$, significant difference in comparison with non-treated neurons (control). $\phi \phi \phi p<0.001$, significant difference in comparison with Li (1 mM)-treated neurons.

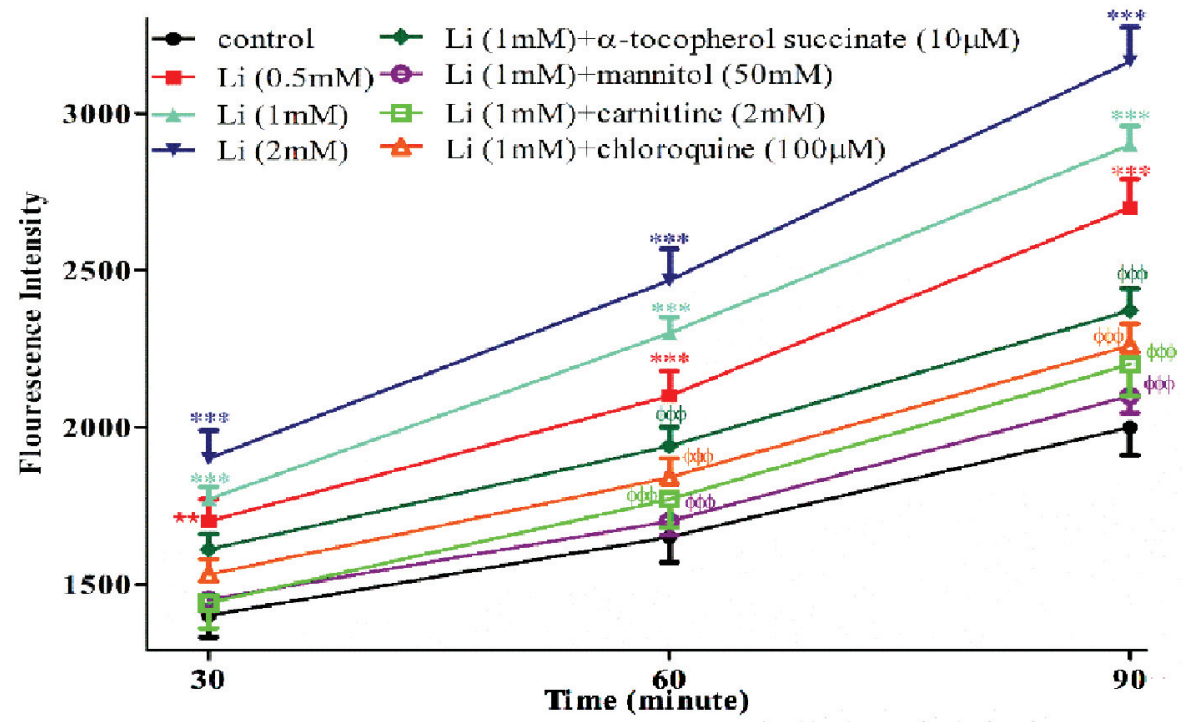

Figure 2: Effect of different concentrations of Li on ROS formation using primary neuronal cortical culture. Reactive oxygen specious were determined spectrofluorometrically by the measurement of highly florescent DCF. Values are shown as mean \pm SD of three separate experiments $(n=3) .{ }^{* \star} p<0.01,{ }^{* \star *} p<0.001$, significant difference in comparison with non-treated neurons (control). $\phi \phi \phi p<0.001$, significant difference in comparison with Li (1 mM)-treated neurons.

GSH depletion could be ascribe to the dismissal of GSSG (Pourahmad et al., 2011).

\section{Discussion}

The results of this study revealed that $50 \%$ of the neural cells were lysed at a concentration of $1 \mathrm{mmol} / \mathrm{L}$
(IC50). In addition, Li significantly increased the ROS formation at EC50 concentration in neuronal cortical culture. Furthermore, a significant amount $(p<0.05)$ of TBARS formed in the third hour of incubation because of Li-induced lipid peroxidation. Li-induced cytotoxicity, ROS and TBARS generation were prevented by $\alpha$-Tocopherol succinate as lipid antioxidant, mannitol as hydroxyl radical scavenger, carnitine as MPT pore sealing 


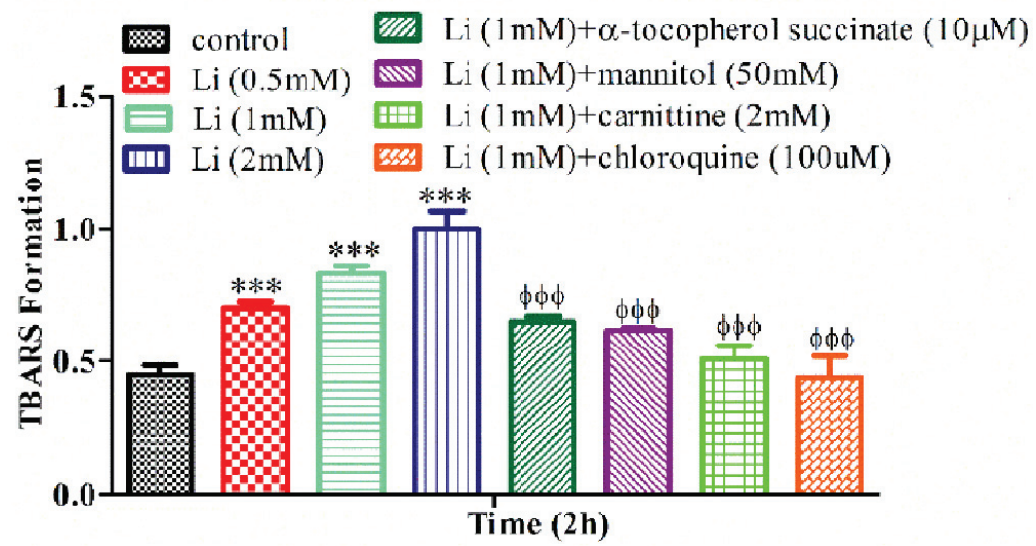

Figure 3: Effect of different concentrations of Li on lipid peroxidation using primary neuronal cortical culture. TBARS formation was measured spectrophotometrically and expressed as $\mu \mathrm{M}$ concentrations. Values are shown as mean $\pm \mathrm{SD}$ of three separate experiments $(n=3) .{ }^{* \star *} p<0.001$, significant difference in comparison with non-treated neurons (control). $\phi \phi \phi p<0.001$, significant difference in comparison with Li (1 mM)-treated neurons.

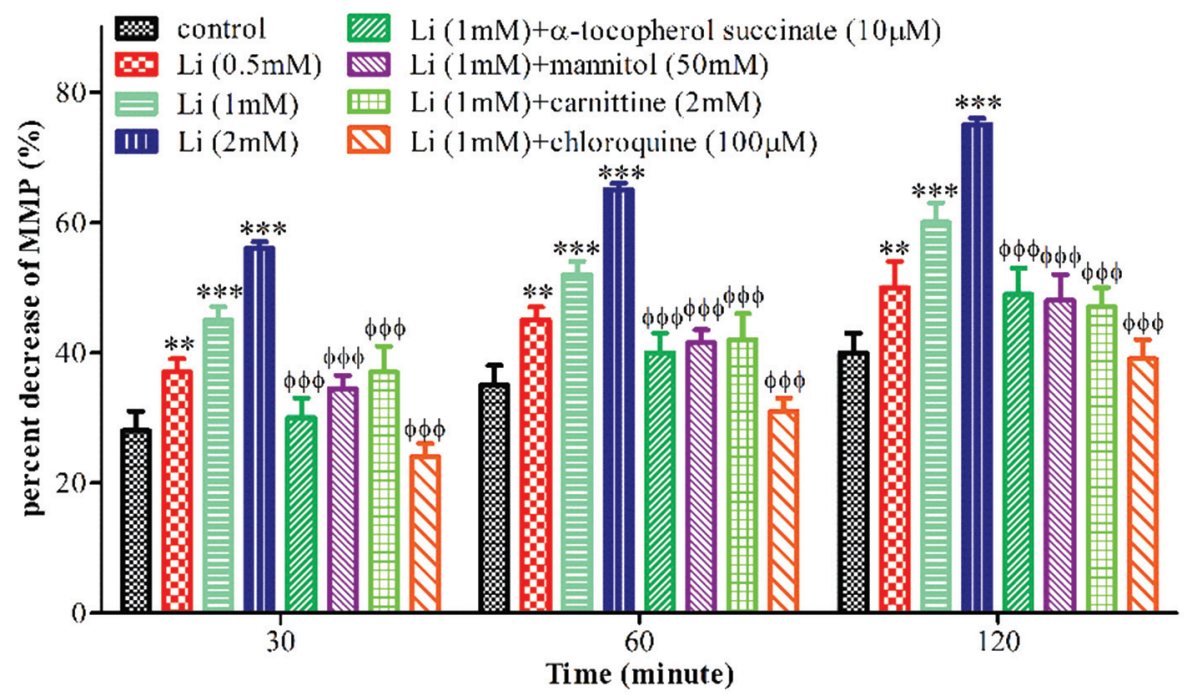

Figure 4: Effect of different concentrations of Li on MMP colapse using primary neuronal cortical culture. The difference in mitochondrial uptake of the rhodamine 123 between the untreated control and Li treated cells is the biochemical basis for the measurement of the percentage of MMP decline. Values are shown as mean \pm SD of three separate experiments $(n=3) .{ }^{* *} p<0.01$, ${ }^{\star \star *} p<0.001$, significant difference in comparison with non-treated neurons (control). $\phi \phi \phi p<0.001$, significant difference in comparison with Li (1 mM)-treated neurons.

agent and chloroquine as lysosomotropic agent. Previous studies demonstrated that chemicals and metals, as well as some medications, which stimulate ATPases, could inhibit lipid peroxidation. It seems that there are close relationships between the stimulation of ATPase activities and inhibition of lipid peroxidation in cerebral cortex synaptosomes (Gilbert and Sawas, 1983; Sawas and Gilbert, 1984).

Li causes mitochondrial membrane potential collapse that was prevented by radical scavengers (mannitol) and lipid antioxidants ( $\alpha$-Tocopherol succinate). This indicates that the decline of mitochondrial membrane potential is a consequence of ROS formation and lipid peroxidation. One of the most important organelles, which play an exclusive role in neuronal cell survival or death, is mitochondria. Mitochondria supply energy for metabolism in the neurons (Clark and Nicklas, 1970). They are responsible for more than $90 \%$ of the cellular ATP generation. Mitochondria also regulate cellular death pathways (Santos et al., 2010). It is thought that mitochondrial dysfunction causes increased production of ROS through damaged respiratory chain, increased lipid peroxidation and impairment of beta- 


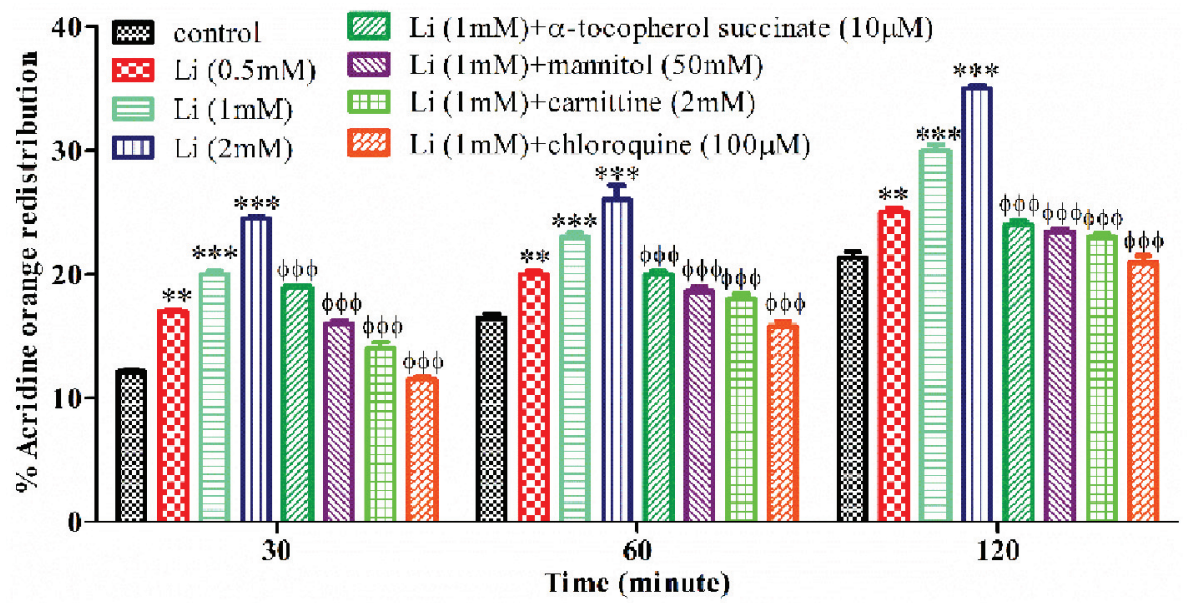

Figure 5: Effect of different concentrations of Li on lysosomal membrane degradation using primary neuronal cortical culture. The redistribution of acridine orange from lysosomes into cytosol in acridine orange loaded neurons was assigned as a biochemical basis for the measurement of lysosomal membrane injury. Highly florescent acridine orange redistribution was determined spectrofluorometrically in treated neurons and shown as the percentage of neurons lysosomal membrane leakage in all groups in three different time intervals.Values are shown as mean $\pm S D$ of three separate experiments $(n=3) .{ }^{\star *} p<0.01,{ }^{\star * *} p<0.001$, significant difference in comparison with nontreated neurons (control). $\phi \phi \phi p<0.001$, significant difference in comparison with Li (1 mM)-treated neurons.

oxidation. This can trigger the release of pro-apoptotic (TNF- $\alpha$ ) and pro-fibrotic (TGF- $\beta$ ) cytokines by Kupffer cells leading to cell death, inflammation and fibrosis (Sepahi et al., 2016). In the previous studies, the effect of Li on isolated heart mitochondria was evaluated. Li increased mitochondrial dysfunction by increasing ROS formation and lipid membrane peroxidation. Li also inhibited the activity of succinate dehydrogenase and reduced ATP production in mitochondria (Salimi et al., 2017a). There are medications that induce ROS formation by increasing $\mathrm{Fe}^{2+}$ accumulation or redox cycling (Castro et al., 2011). This may cause mitochondrial nitrative and/or oxidative changes as a triggering factor for harmful alteration, such as unfolding, aggregation, and fragmentation of proteins or catalytic enzymes. All promoting degradations of modified proteins lead to autophagy and/or mitophagy (Bayeva et al., 2013). Damages to mitochondria can lead to necrotic/apoptotic cellular and neuronal damages; therefore, the MPT pore sealing agents such as carnitine potentially reduces ROS formation and neural deaths.

Li-induced AO release was also prevented by hydroxyl radical scavengers (mannitol), lipid antioxidant ( $\alpha$-Tocopherol succinate), and MPT pore sealing agent (carnitine). This means that any damage to the lysosomal membrane of nerve cells occurs due to increased ROS and TBARS formation. The results of this study revealed that Li induced lysosomal membrane damage leading to protein degradation, lipid peroxidation, and mitochondrial dysfunction. This is similar to the damages caused by the formation of intracellular ROS such as $\mathrm{H}_{2} \mathrm{O}_{2}$, as a result of CYP450 mediated metabolic activation (Razavi-
Azarkhiavi et al., 2014; Seglen and Gordon, 1982). $\mathrm{H}_{2} \mathrm{O}_{2}$ is one of the most abundant and stable ROS molecules in organisms. $\mathrm{H}_{2} \mathrm{O}_{2}$ has an intermediate oxidation number $(-1)$ so it can possess reducing and oxidizing properties that are important for its cellular multi-functionality. $\mathrm{H}_{2} \mathrm{O}_{2}$ can directly or indirectly (via the formation of other ROS) contribute to lipid peroxidation, protein carbonylation, DNA and the oxidation of methionine residues and thiol groups. $\mathrm{H}_{2} \mathrm{O}_{2}$ acts as a signaling molecule in various cellular processes (Imlay, 2003). Specific aquaporins isoforms facilitate the diffusion of $\mathrm{H}_{2} \mathrm{O}_{2}$ across biological membranes and, therefore, crucially impact on the membrane permeability of $\mathrm{H}_{2} \mathrm{O}_{2}$ (Bienert and Chaumont, 2014). Hydroxyl radical damages the lysosomal membrane and consequently digestive enzymes (cathepsins) enter the plasma. Cathepsins could target the mitochondrial outer membranes or activate Bid, Bax, and other lytic enzymes including phospholipase $\mathrm{A}_{2}$ to open the MPT pores. This leads to the release of cytochrome $\mathrm{c}$ and the collapse of mitochondrial inner membrane potential. Cytochrome $\mathrm{c}$ release cause further oxidative stress induction through increasing mitochondrial $\mathrm{H}_{2} \mathrm{O}_{2}$ diffusion into the lysosomes and generating more lysosomal hydroxyl radicals (Pourahmad et al., 2012; Salimi et al., 2019). Therefore, we conclude that mitochondrial and lysosomal damages are interdependent and any damage to mitochondria ultimately causes damage to lysosome and vice versa. Therefore, this is the reason why lysosomotropic agents (chloroquine) prevents mitochondrial damage. On the other hand, carnitine as MPT pore sealing agents prevented damage to the lysosomes 


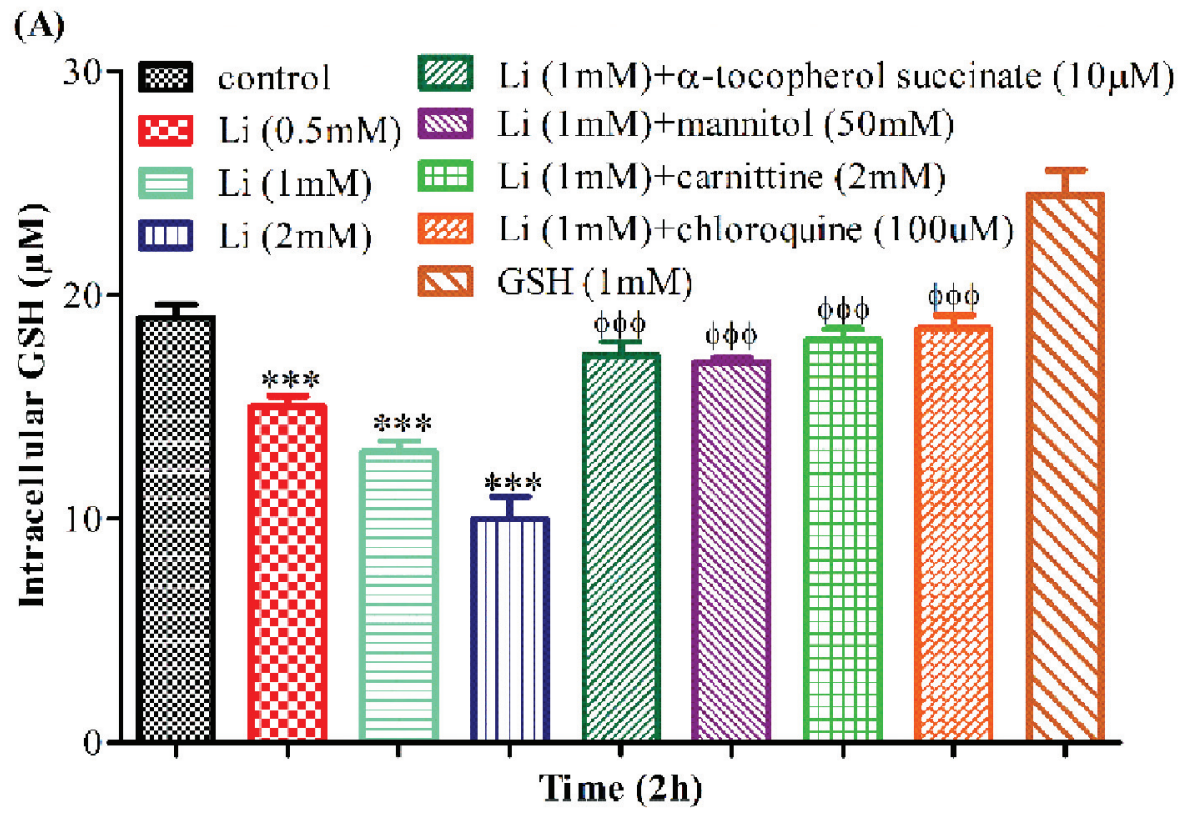

(B)

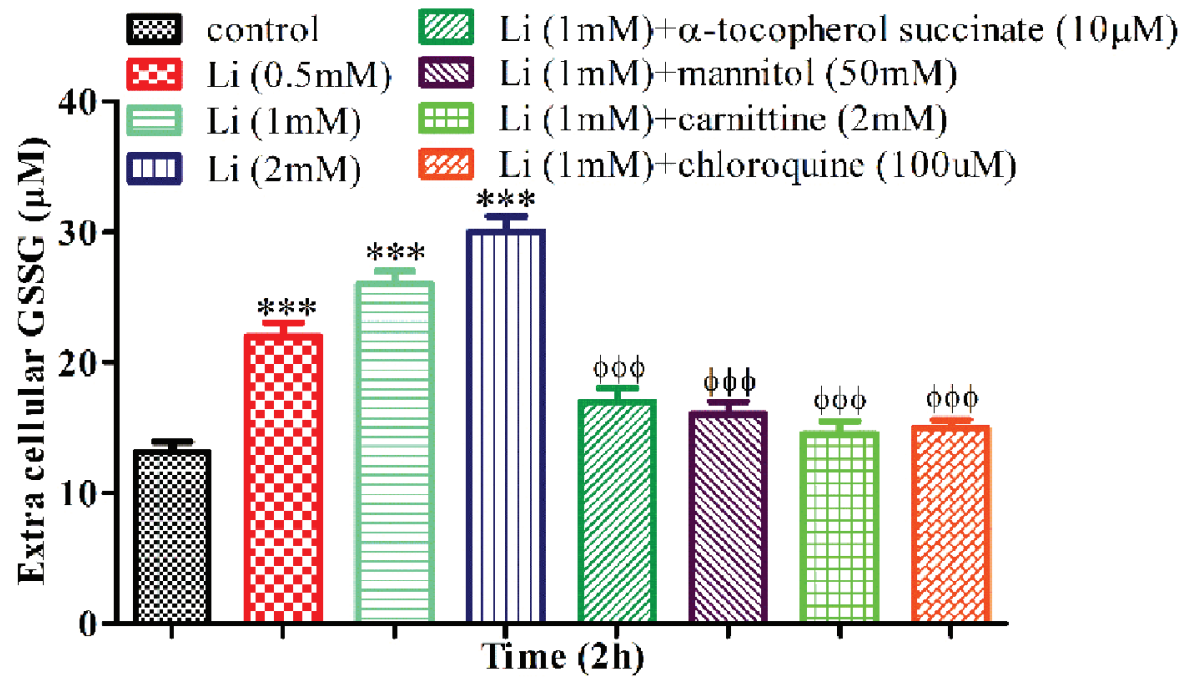

Figure 6: Effect of different concentrations of Li onGSH depletion and the net values of GSH (a) and GSSG (b) using primary neuronal cortical culture. Intracellular GSH and extra cellular GSSG were measured spectrofluorometrically. Values are shown as mean \pm SD of three separate experiments $(n=3)$. ${ }^{* \star *} p<0.001$, significant difference in comparison with non-treated neurons (control). $\phi \phi \phi p<0.001$, significant difference in comparison with Li (1 mM)-treated neurons.

The incubation of isolated neurons with Li induced intracellular GSH depletion. Most of the GSH depletion could be justified to the efflux of GSSG. In this test as in previous experiments intracellular GSH depletion and increased extracellular GSSG prevented by lipid antioxidant ( $\alpha$-Tocopherol), hydroxyl radical scavenger (mannitol), MPT pore sealing agent (carnitine), and lysosomotropic agent (chloroquine). These results suggested that neural cortical cells would be survived by reducing ROS and TBARS production or protecting neural lysosomes and mitochondria. GSH is an important intracellular antioxidant, which plays a critical role in maintaining cellular redox homeostasis (Lash, 2006). It is a non-enzymatic thiol antioxidant capable of protecting cells against oxidative stress (Razavi-Azarkhiavi et al., 2016). When isolated neurons were incubated with $\mathrm{Li}$, glutathione depletion occurred due to ROS generation. Results of this study demonstrated that depleting of isolated neural GSH, potentiated all Li induced ROS generation, mitochondrial membrane potential collapse, 
lysosomal membrane leakiness, and neural cytotoxicity. Therefore, it is confirmed that the cytotoxic mechanism of Li is mediated through oxidative stress.

\section{Conclusion}

Our results propose that add-on therapy or nutritional consumption of antioxidant agents (especially antioxidants that protects lysosomes and mitochondria) could reduce the chance of Li-induced neurotoxicity.

Acknowledgements: The data provided in this article was extracted from the Pharm. D. thesis of Dr. Romina Askian. The thesis was conducted under supervision of Prof. Jalal Pourahmad at Department of Toxicology and Pharmacology, Faculty of Pharmacy, Shahid Beheshti University of Medical Sciences, Tehran, Iran.

Disclosure statement: The authors declare that they have no conflicts of interest.

\section{References}

Arraf Z., Amit T., Youdim M.B., Farah R., Lithium and oxidative stress lessons from the MPTP model of Parkinson's disease. Neurosci. Lett., 2012, 516(1), 57-61.

Baradaran Rahimi V., Askari V.R., Hosseini M., Yousefsani B.S., Sadeghnia H.R., Anticonvulsant Activity of Viola tricolor against Seizures Induced by Pentylenetetrazol and Maximal Electroshock in Mice. Iran. J. Med. Sci., 2019, 44(3), 220-226.

Bayeva M., Gheorghiade M., Ardehali H., Mitochondria as a therapeutic target in heart failure. J. Am. Coll. Cardiol., 2013, 61(6), 599-610.

Bienert G.P., Chaumont F., Aquaporin-facilitated transmembrane diffusion of hydrogen peroxide. Biochim. Biophys. Acta, 2014, 1840(5), 1596-1604.

Castro L., Demicheli V., Tortora V., Radi R., Mitochondrial protein tyrosine nitration. Free Radic. Res., 2011, 45(1), 37-52.

Clark J.B., Nicklas W.J., The metabolism of rat brain mitochondria. Preparation and characterization. J. Biol. Chem., 1970, 245(18), 4724-4731.

Clausen N.G., Hansen T.G., Disma N., Anesthesia Neurotoxicity in the Developing Brain: Basic Studies Relevant for Neonatal or Perinatal Medicine. J. Perinatol., 2019, 46(4), 647-656.

Costa G., De Luca, M.A., Piras G., Marongiu J., Fattore L., Simola N., Neuronal and peripheral damages induced by synthetic psychoactive substances: an update of recent findings from human and animal studies., Neural. Regen. Res., 2020, 15(5), 802-816.

De Cates A.N., Morlet J., Antoun Reyad A., Tadros G., Lithium overdose and delayed severe neurotoxicity: timing for renal replacement therapy and restarting of lithium. BMJ Case Rep., 2017, 1-3.
Decker B.S., Goldfarb D.S., Dargan P.I., Friesen M., Gosselin S., Hohhman R.S., et al., Extracorporeal Treatment for Lithium Poisoning: Systematic Review and Recommendations from the EXTRIP Workgroup. Clin. J. Am. Soc. Nephrol., 2015, 10(5), 875-887.

Donaldson I.M., Cuningham J., Persisting neurologic sequelae of lithium carbonate therapy. Arch. Neurol., 1983, 40(12), 747-751.

Efendiev A.M. Kerimov B.F., The effect of starvation of lipid peroxidation in synaptosomal and mitochondrial factions of various brain structures. Vopr. Med. Khim., 1994, 40(2), 34-37.

Ferraro S.A., Domingo M.G., Etcheverrito A., Olmedo D.G., Tasat D.R., Neurotoxicity mediated by oxidative stress caused by titanium dioxide nanoparticles in human neuroblastoma (SH-SY5Y) cells. J. Trace Elem. Med. Bio., 2020, 57, 116-123.

Garza-Lombo C., Pappa A., Panayiotidis M.I., Gonsebatt M.E. Franco R., Arsenic-induced neurotoxicity: a mechanistic appraisal. J. Biol. Inorg. Chem., 2019, 24(8), 1305-1316.

Gilbert J.C., Sawas A.H., ATPase activities and lipid peroxidation in rat cerebral cortex synaptosomes. Arch. Int. Pharmacod. T., 1983, 263(2), 189-196.

Hampel H., Lista S., Mango D., Nisticò R., Perry G., Avila J., et al., Lithium as a Treatment for Alzheimer's Disease: The Systems Pharmacology Perspective. J. Alzheimers Dis., 2019, 69(3), 615-629.

Hancock P.A., Vasmatzidis I., Effects of heat stress on cognitive performance: the current state of knowledge. Int. J. Hyperther., 2003, 19(3), 355-372.

Hansen H.E., Amdisen A., Lithium intoxication. (Report of 23 cases and review of 100 cases from the literature). Med. Anthropol. Q., 1978, 47(186), 123-144.

Hissin P.J., Hilf R., A fluorometric method for determination of oxidized and reduced glutathione in tissues. Anal. Biochem., 1976, 74(1), 214-226.

Horta E., Bongiorno C., Ezzeddine M., Neil E.C., Neurotoxicity of antibodies in cancer therapy: A review. Clin. Neurol. Neurosurg., 2020, 188, 105566-105574.

Huang X., Li N., Pu Y., Zhang T., Wang B., Neuroprotective Effects of Ginseng Phytochemicals: Recent Perspectives. Molecules, 2019, 24(16), 2939-2959.

Imlay J.A., Pathways of oxidative damage. Annu. Rev. Microbiol., 2003, 57, 395-418.

Ji C., Ni Q., Chen W., Yang Z., Ma D., General anesthetic neurotoxicity in the young: Mechanism and prevention. Neurosci. Biobehav. Rev., 2019, 107, 883-896.

Khansari M.R., Yousefsani B.S., Kobarfard F., Faizi M., Pourahmad J., In vitro toxicity of perfluorooctane sulfonate on rat liver hepatocytes: probability of distructive binding to CYP 2E1 and involvement of cellular proteolysis. Environ. Sci. Pollut. Res. Int., 2017, 24(29), 23382-23388.

Kiani A., Yousefsani B.S., Doroudian P., Seydi E., Pourahmad J., The mechanism of hepatotoxic effects of sodium nitrite on isolated rat hepatocytes. J. Toxicol. Env. Health Sci., 2017, 9(3), 244-250.

Kordafshari G., Shams Ardakani M.R., Keshavarz M., Esfahani M.M., Nazem E., Moghimi M., et al., The Role of Phlebotomy (Fasd) and Wet Cupping (Hijamat) to Manage Dizziness and Vertigo From the Viewpoint of Persian Medicine. Evid.-Based Compl. Alt., 2017, 22(3), 369-373.

Lang E.J., Davis S.M., Lithium neurotoxicity: the development of irreversible neurological impairment despite standard 
monitoring of serum lithium levels. J. Clin. Neurosci., 2002, 9(3), 308-317.

Lash L.H., Mitochondrial glutathione transport: physiological, pathological and toxicological implications. Chem. Biol. Interact., 2006, 163(1-2), 54-67.

Malhotra S., Sharma S., Awasthi A., Sharma B.K., Cerebellar damage in a patient on lithium developing enteric Fever : a case report. Indian. J. Psychiat., 1985, 27(3), 255-258.

Mani J., Tandel S.V., Shah P.U., Karnad D.R., Prolonged neurological sequelae after combination treatment with lithium and antipsychotic drugs. J. Neurol. Neurosur. Ps., 1996, 60(3), 350-351.

Mansoori P., Akhondzadeh S., Raisi F., Ghaeli P., Jamshidi A.H., Nasehi A.A., et al., A Randomized, Double-blind, Placebo-controlled Study of Safety of the Adjunctive Saffron on Sexual Dysfunction Induced by a Selective Serotonin Reuptake Inhibitor. J. Med. Plants Res., 2011, 10(37), 1-10.

Meberg P.J., Miller M.W., Culturing hippocampal and cortical neurons. Method. Cell Biol., 2003, 71, 111-127.

Megarbane B., HanakA.S., Chevillard L., Lithium-related neurotoxicity despite serum concentrations in the therapeutic range: risk factors and diagnosis. Shanghai Arch. Psychiat., 2014, 26(4), 243-244.

Mesquita J., Cepa S., Silva L., Machado A., Lithium neurotoxicity at normal serum levels. J. Neuropsych. Clin. N., 2010, 22(4), 451-p e29-451 e29.

Pourahmad J., Eskandari M.R., Kaghazi A., Shaki F., Shahraki J., Kobarfard J., A new approach on valproic acid induced hepatotoxicity: involvement of lysosomal membrane leakiness and cellular proteolysis. Toxicol. In Vitro, 2012, 26(4), 545-551.

Pourahmad J., Mortada Y., Eskandari M.R., Shahraki J., Involvement of Lysosomal Labilisation and Lysosomal/mitochondrial Cross-Talk in Diclofenac Induced Hepatotoxicity. Iran. J. Pharm. Res., 2011, 10(4), 877-887.

Pourahmad J., O’Brien P.J., A comparison of hepatocyte cytotoxic mechanisms for $\mathrm{Cu}^{2+}$ and $\mathrm{Cd}^{2+}$. Toxicology, 2000, 143(3), 263-273.

Pourahmad J., Rabiei M., Jokar F., O'Brien P.J., A comparison of hepatocyte cytotoxic mechanisms for chromate and arsenite. Toxicology, 2005, 206(3), 449-460.

Razavi-Azarkhiavi K., Behravan J., Mosaffa F., Sehatbakhsh S., Shirani K., Karimi G., Protective effects of aqueous and ethanol extracts of rosemary on $\mathrm{H}_{2} \mathrm{O}_{2}$-induced oxidative DNA damage in human lymphocytes by comet assay. J. Complement. Integr. Med., 2014, 11(1), 27-33.

Razavi-Azarkhiavi K., Jafarian A.H., Abnous K., Razavi B.M., Shirani K., Zeinali M., et al., The Comparison of Biodistribution, Efficacy and Toxicity of Two PEGylated Liposomal Doxorubicin Formulations in Mice Bearing C-26 Colon Carcinoma: a Preclinical Study. Drug Res., 2016, 66(06), 330-336.

Richardson J.R., Fitsanakis V., Westerink R.H.S., Kanthasamy A.G., Neurotoxicity of pesticides. Acta Neuropathol., 2019, 138(3), 343-362.

Salimi A., Gholamifar E., Naserzadeh P., Hosseini M.J., Pourahmad J., Toxicity of lithium on isolated heart mitochondria and cardiomyocyte: A justification for its cardiotoxic adverse effect. J. Biochem. Mol. Toxic., 2017a, 31(2), 1-8.

Salimi A., Paeezi M., Yousefsani B.S., Shadnia S., HassanianMoghaddam H., Zamani N., et al., Inhibition of glucose-6-phosphate dehydrogenase protects hepatocytes from aluminum phosphide-induced toxicity. Pestic. Biochem. Phys., 2017b, 143, 141-146.

Salimi A., Pirhadi R., Jamali Z., Ramazani M., Yousefsani B.S., Pourahmad J., Mitochondrial and lysosomal protective agents ameliorate cytotoxicity and oxidative stress induced by cyclophosphamide and methotrexate in human blood lymphocytes. Hum. Exp. Toxicol., 2019, 38(11), 1266-1274.

Salimi A., Roudkenar M.H., Sadeghi L., Mohseni A., Seydi E., Pirahmadi N., et al., Ellagic acid, a polyphenolic compound, selectively induces ROS-mediated apoptosis in cancerous B-lymphocytes of CLL patients by directly targeting mitochondria. Redox Biol., 2015, 6, 461-471.

Santos R.X., Correia S.C., Wang X., Perry G., Smith M.A., Moreira P.I., et al., Alzheimer's disease: diverse aspects of mitochondrial malfunctioning. Int. J. Clin. Exp. Pathol., 2010, 3(6), 570-581.

Sawas A.H., Gilbert J.C., Possible mechanism of inhibition by lipid peroxidation of ATPase activities of rat cerebral cortex synaptosomes. Arch. Int. Pharmacod. T., 1984, 269(1), 4-11.

Sawas A.H., Gilbert J.C., Lipid peroxidation as a possible mechanism for the neurotoxic and nephrotoxic effects of a combination of lithium carbonate and haloperidol. Arch. Int. Pharmacod. T., 1985, 276(2), 301-312.

Sawas A.H., Gilbert J.C., Watson M.E., Effects of lithium salts on lipid peroxidation activity of synaptosomes and kidney homogenates. Arch. Int. Pharmacod. T., 1986, 281(2), 192-197.

Schou M., Amdisen A., Trap-Jensen J., Lithium poisoning. Am. J. Psychiat., 1968, 125(4), 520-527.

Seglen P.O., Gordon P.B., 3-Methyladenine: specific inhibitor of autophagic/lysosomal protein degradation in isolated rat hepatocytes. Proc. Natl. Acad. Sci. U.S.A., 1982, 79(6), 1889-1892.

Sepahi S., Jafarian-Dehkordi A., Mirlohi M., Shirani K., Etebari M., Protective role of Lactobacillus plantarum A7 against irinotecaninduced genotoxicity. Avicenna. J. Phytomed., 2016, 6(3), 329-335.

Shen H.M., Shi C.Y., Shen Y., Ong C.N., Detection of elevated reactive oxygen species level in cultured rat hepatocytes treated with aflatoxin B1. Free Radical. Bio. Med., 1996, 21(2), 139-146.

Sohrabvand F., Mahroozade S., Bioos S., Nazari S.M., Dabaghian F.H., Improvement in Sperm Parameters With Traditional Iranian Remedy: A Case Report. Evid.-Based Compl. Alt., 2017, 22(2), 223-226.

Soni S., Lithium neurotoxicity presenting as dementia with therapeutic serum lithium levels. BMJ Case Rep., 2019, 12(1), 1-3.

Soriano-Barcelo J., Alonso M.T., Traba M.B., Vilar, A.A., Kahn D.A., A case with reversible neurotoxicity after 2 years of dementia secondary to maintenance lithium treatment. J. Psychiatr. Pract., 2015, 21(2), 154-159.

Staff N.P., Cavaletti G., Islam B., Lustberg M., Psimaras D., Tamburin S., Platinum-induced peripheral neurotoxicity: From pathogenesis to treatment. J. Peripher. Nerv. Syst., 2019, 24(Suppl.2), S26-S39.

Swartz C.M., Jones P., Hyperlithemia correction and persistent delirium. J. Clin. Pharmacol., 1994, 34(8), 865-870.

Taranova N.P., Nilova N.S., Lipid peroxidation by rat brain synaptosomes in sleep disorders. Fiziol. Zh. SSSR. Im. I. M. Sechenova., 1986, 72(8): 1065-1068.

Vodovar D., Megarbane B., Defining predictive factors of severity and indications for extracorporeal toxin removal in lithium 
poisoning: not an easy objective. Clin. Toxicol., 2017, 55(1), 69-70.

Yao C.J., Lin C.W., Lin-Shiau S.Y., Altered intracellular calcium level in association with neuronal death induced by lithium chloride. J. Formos. Med. Assoc., 1999, 98(12), 820-826.

Yousefsani B.S., Mehri S., Pourahmad J., Hosseinzadeh H., Crocin Prevents Sub-Cellular Organelle Damage, Proteolysis and Apoptosis in Rat Hepatocytes: A Justification for Its Hepatoprotection. Iran. J. Pharm. Res., 2018, 17(2), 553-562.

Yousefsani B.S., Mohajeri S.A., Moshiri M., Jafarian A.H., Hosseinzadeh H., Effect of Intravenous Lipid Emulsion on
Clozapine Acute Toxicity in Rats. J. Pharmacopuncture., 2019, 22(3), 147-153.

Yousefsani B.S., Akbarizadeh N., Pourahmad J., The antioxidant and neuroprotective effects of Zolpidem on acrylamide-induced neurotoxicity using Wistar rat primary neuronal cortical culture. Tox. Rep., 2020, 7, 233-240.

Zhang H., Yang X., Li X., Cheng Y., Zhang H., Chang l., et al., Oxidative and nitrosative stress in the neurotoxicity of polybrominated diphenyl ether-153: possible mechanism and potential targeted intervention. Chemosphere, 24(Suppl.2), S26-S39. 